\title{
El ovario poliquístico y las enfermedades dermatológicas
}

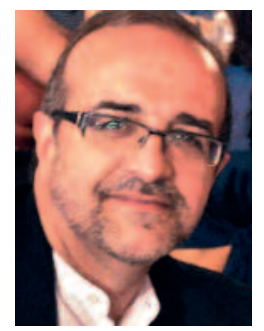

Jesús S. Jiménez López

Jefe de la Sección de Ginecología. Servicio de Obstetricia y Ginecología.

Hospital Universitario 12 Octubre. Madrid.

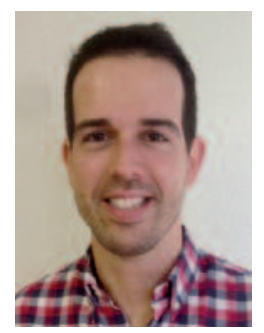

\section{Alejandro Olloqui Escalona}

Médico adjunto. Servicio de Obstetricia y Ginecología. Hospital Universitario 12 Octubre. Madrid.

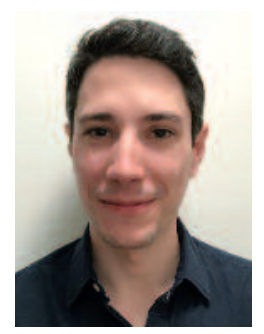

Álvaro Díez Álvarez

Médico adjunto.

Servicio de Obstetricia y

Ginecología.

Hospital Universitario 12 Octubre.

Madrid.

\section{RESUMEN}

El síndrome del ovario poliquístico (SOP) es una enfermedad multisistémica, que se caracteriza por disfunción ovulatoria e hiperandrogenismo. Su diagnóstico se basa en los criterios de Rotterdam, y afecta a entre un 6,5 y un $8 \%$ de la población. Su patogenia está determinada por una secreción aumentada de hormona luteinizante, que conduce a un aumento de los andrógenos de origen ovárico, a lo cual se une un estado hiperinsulinémico, que disminuye la síntesis de globulina de fijación a las hormonas sexuales o SHBG (sex hormone-binding globulin) y potencia la síntesis de andrógenos. A nivel dermatológico, los signos con los que se relaciona son hirsutismo, acné, acantosis nigricans y alopecia.

El tratamiento de los trastornos dermatológicos generados por el SOP comprende tanto las medidas terapéuticas locales (p. ej., depilación permanente o definitiva) como las sistémicas. De entre los tratamientos sistémicos, destacamos los anticonceptivos hormonales orales, así como los fármacos antiandrogénicos; ambos grupos de fármacos han demostrado mejorar el acné y el hirsutismo de estas pacientes. Para el tratamiento de la alopecia, el minoxidil al 2 o al $5 \%$ y la finasterida han resultado efectivos. En todos los grupos de pacientes, se recomienda dieta, ejercicio y cambios en el estilo de vida.

Palabras clave: ovario poliquístico, hiperandrogenismo, síndrome metabólico, hirsutismo, acné.

\section{ABSTRACT \\ Polycystic ovary syndrome (PCOS) is a multisystemic dis- ease. Oligo-anovulation and hyperandrogenism are the most important features of this syndrome. Diagnosis of PCOS is made according to the Rotterdam criteria, and the syn- drome affects between 6.5 and $8 \%$ of the female popula- tion. Pathogenically, PCOS patients show an increased luteinizing hormone secretion, that generates an increased secretion of androgens, and a hyperinsulinemic state, that}


also contributes to this hyperandrogenism by increasing the androgen secretion and diminishing sex hormone-binding globulin (SHBG). Dermatologic problems that occur frequently in PCOS patients include hirsutism, acne, alopecia and acanthosis nigricans.

Treatment of dermatologic symptoms of PCOS include hormonal contraceptives and antiandrogenic drugs, that have shown an improvement in hirsutism and acne. Local therapies like depilation are also useful to treat some of the symptoms. Alopecia can be treated with minoxidil 2 or $5 \%$ or finasteride. Lifestyle changes are recommended in this group of patients, especially in obese or overweight patients.

Keywords: polycystic ovary, hyperandrogenism, metabolic syndrome, hirsutism, acne.

\section{INTRODUCCIÓN}

El síndrome del ovario poliquístico (SOP), descrito por Irving Stein y Michel Leventhal en $1935^{1}$ -y, por ello, también conocido como síndrome de Stein-Leventhal-, es una enfermedad compleja que cursa con signos y síntomas variados. Se trata de la causa más común de infertilidad en las mujeres ${ }^{2}$, se manifiesta frecuentemente durante la adolescencia y se caracteriza por disfunción ovulatoria e hiperandrogenismo.

Es un síndrome heterogéneo tanto en su clínica como en los hallazgos bioquímicos; de hecho, durante muchos años, ha existido el debate sobre si el SOP representa una sola enfermedad o, por el contrario, se trata de múltiples enfermedades con fenotipos solapados entre sí.

El diagnóstico actual se basa en los criterios de Rotterdam y se define por la presencia de, al menos, dos de los siguientes hallazgos: oligomenorrea/amenorrea, hiperandrogenismo y ovarios poliquísticos (diagnosticado por ecografía) (tabla 1).

En función de los criterios descritos en el Consenso de Rotterdam (2003), podemos distinguir cuatro fenotipos de SOP:

- Fenotipo 1: hiperandrogenismo, oligoovulación/anovulación y ovarios poliquísticos.

- Fenotipo 2: hiperandrogenismo con oligoovulación/anovulación.
Tabla 1. Criterios de Rotterdam, 2003 (2 de los 3)

1. Oligomenorrea/amenorrea

2. Hiperandrogenismo clínico (acné, alopecia, hirsutismo) o analítico

3. Hallazgo ecográfico de ovarios poliquísticos

*Todo ello excluyendo otras causas de hiperandrogenismo y anovulación como el síndrome de Cushing, los tumores secretores de andrógenos o la hiperplasia suprarrenal congénita.

- Fenotipo 3: hiperandrogenismo con ovario poliquístico (ovulaciones normales).

- Fenotipo 4: oligoovulación/anovulación con ovario poliquístico (sin hiperandrogenismo).

\section{PATOGENIA}

El SOP es un síndrome que afecta a entre el 6,5 y el $8 \%$ de las mujeres, aunque, en función de las series, la prevalencia se sitúa en entre el 5 y el $22 \%$. Se ha sugerido una predisposición a manifestar el SOP adquirida durante la etapa fetal por la exposición a andrógenos.

La patogenia del SOP es aún desconocida, sin embargo, varias de sus características se conocen mejor. El ovario parece ser el principal responsable del exceso de producción de andrógenos ${ }^{3}$. Se ha objetivado una disfunción hipotalámica en el 
SOP, que hace que la frecuencia con la que el hipotálamo segrega la hormona liberadora de gonadotropinas (GnRH) se vea aumentada, lo cual genera una secreción preferencial de la hormona luteinizante (LH) sobre la hormona foliculoestimulante (FSH) por parte de la hipófisis ${ }^{4}$. La LH estimula las células de la teca del ovario que segregan andrógenos, fundamentalmente, androstenodiona, que será posteriormente convertida a testosterona o bien aromatizada en las células de la granulosa y convertida en estrógenos. La presencia de este ambiente de hiperandrogenismo desempeña un papel relevante en la oligoovulación/anovulación propia de estas pacientes. Por último, las pacientes con SOP tienen una tendencia a la resistencia a la insulina, lo que genera un estado hiperinsulinémico, que todavía se agrava más en muchas pacientes debido a la obesidad que presentan, y que estimula la síntesis de andrógenos en las células de la teca, además de inhibir la síntesis hepática de la globulina de fijación a las hormonas sexuales o SHBG (sex hormone-binding globulin), generando ambos efectos un aumento de la testosterona circulante, que es la testosterona activa ${ }^{5}$.

\section{MANIFESTACIONES SISTÉMICAS DEL SÍNDROME DEL OVARIO POLIQUÍSTICO}

Como hemos comentado en la introducción, la clínica general del SOP es la oligomenorrea y el hiperandrogenismo, así como la presencia de factores de riesgo de enfermedad cardiovascular.

La irregularidad menstrual típica del SOP se inicia en el período peripuberal; típicamente, genera oligomenorrea (menos de nueve menstruaciones en un año) y, menos frecuentemente, amenorrea (tres o más meses sin menstruación).

El hiperandrogenismo propio del SOP puede manifestarse de forma analítica o por la clínica (acné, hirsutismo, alopecia androgénica, etc.). Otros síntomas más graves de virilización como la clitoromegalia, el incremento de la masa mus- cular o el cambio del timbre de la voz nos deben orientar hacia un tumor productor de andrógenos, ovárico, suprarrenal o de otra índole.

Los factores de riesgo de enfermedad cardiovascular que encontramos en estas pacientes se deben a la elevada presencia de sobrepeso y obesidad; entre el 40 y el $80 \%$ de ellas, según las series, presentan, al menos, sobrepeso, y la resistencia a la insulina es muy frecuente en las pacientes con SOP, tanto obesas (hasta un $70 \%$ ) como con normopeso (el 20-30\%). El sobrepeso y la resistencia a la insulina son, además, factores de riesgo de desarrollo de diabetes mellitus de tipo 2 (DM2).

A nivel digestivo, la aparición de esteatohepatitis (inflamación grasa del hígado) no alcohólica es más frecuente en pacientes con SOP que en la población general.

Se ha relacionado también el SOP con trastornos psiquiátricos como la ansiedad o la depresión y con trastornos respiratorios como el síndrome de apneas e hipopneas durante el sueño (SAHS), que son más frecuentes en este tipo de pacientes que en la población general.

En definitiva, el SOP es un síndrome con efectos multisistémicos, a nivel endocrino, reproductivo, cardiovascular, dermatológico, gastrointestinal, pulmonar e, incluso, psiquiátrico.

\section{MANIFESTACIONES DERMATOLÓGICAS DEL SÍNDROME DEL OVARIO POLIQUÍSTICO}

Las manifestaciones dermatológicas del SOP incluyen el hirsutismo, el acné, la piel grasa, la alopecia y la acantosis nigricans.

\section{Hirsutismo}

El hirsutismo se define como el exceso de vello terminal en regiones de localización típicamente masculina, como pueden ser el mentón, el labio superior, la espalda, el abdomen, las aréolas, el espacio intermamario o las mejillas (fig. 1). 


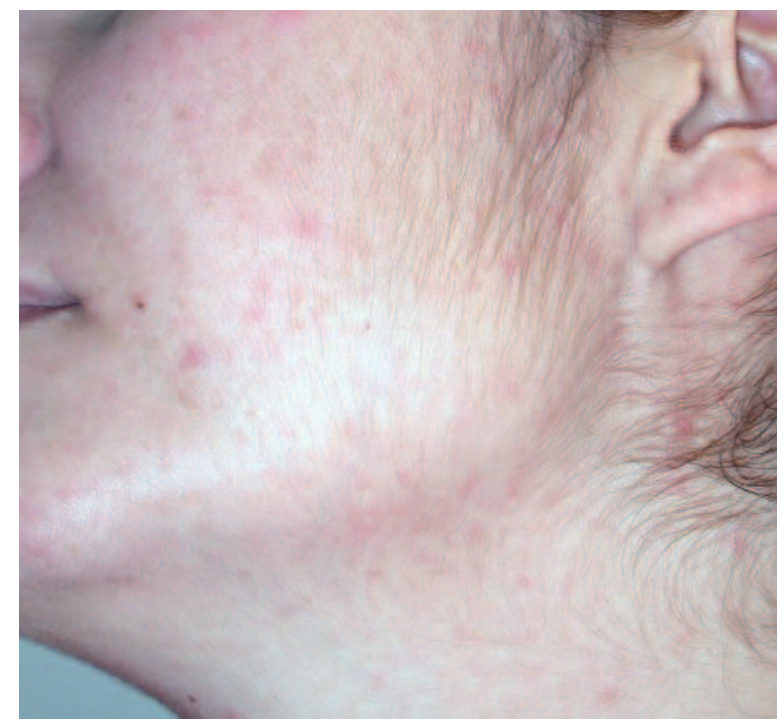

Figura 1. Exceso de vello terminal en regiones de localización típicamente masculina, como puede ser el mentón (por gentileza de la Dra. Aurora Guerra-Tapia).

Se debe a la transformación de vello en vello terminal en áreas del cuerpo que son sensibles a los andrógenos. Hasta el $60 \%$ de las mujeres con SOP presentan hirsutismo. El andrógeno más relacionado con el hirsutismo es la dihidrotestosterona (DHT), que es la forma más activa de la testosterona, sintetizada en las mujeres en la piel por acción de la enzima $5 \alpha$-reductasa. Las mujeres con hirsutismo presentan una mayor actividad de la $5 \alpha$-reductasa a nivel cutáneo. La gravedad del hirsutismo se cuantifica típicamente mediante la escala de Ferriman-Gallwey modificada ${ }^{6}$ (fig. 2).

\section{Acné}

El acné es otro de los trastornos dermatológicos que pueden ocurrir en el SOP, aunque es menos frecuente que el hirsutismo. Se estima que entre el 23 y el $35 \%$ de las pacientes con SOP sufren acné ${ }^{7}$. En comparación con el acné puberal, el de las pacientes con SOP suele presentarse con más cantidad de lesiones inflamatorias en la cara, el cuello, el tórax y la región superior de la espalda. También suele ser más persistente, instaurar-

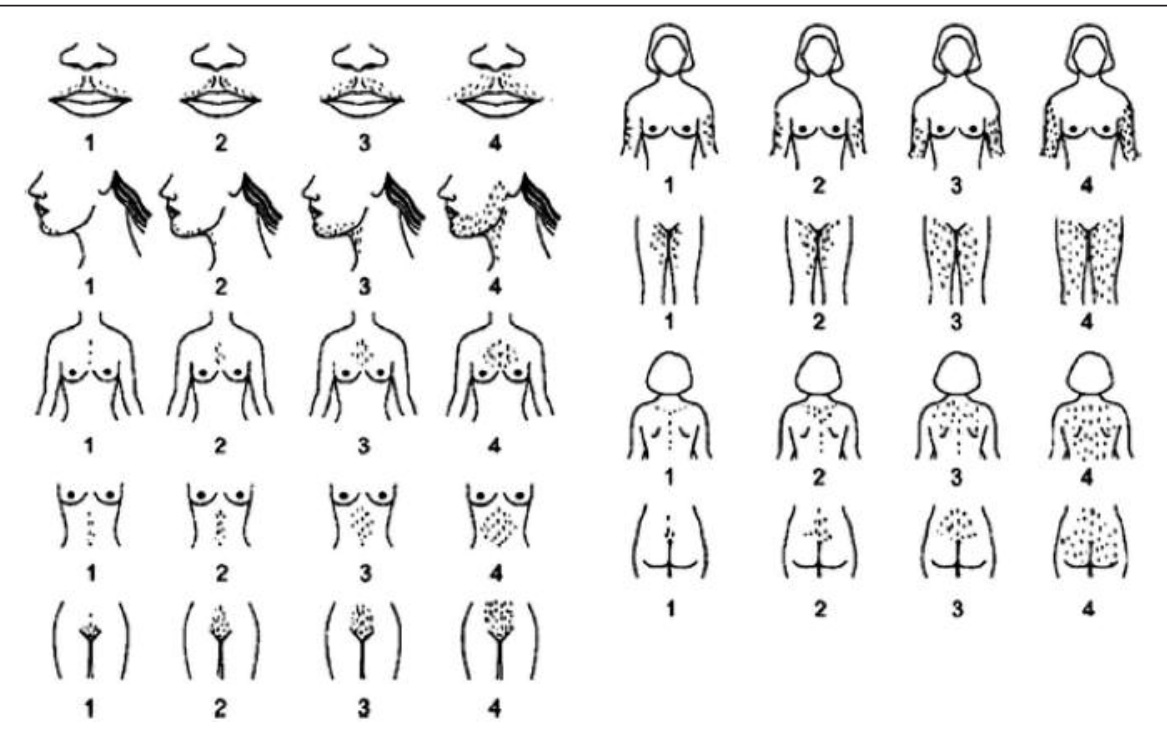

Figura 2. Escala de Ferriman-Gallwey modificada. Nueve regiones corporales (labio superior, mentón, brazos, abdomen superior, abdomen inferior, región superior de la espalda, región inferior de la espalda y muslos) deben ser valoradas según la presencia de vello terminal de acuerdo con: 1 punto (mínima presencia), 2 puntos (superior a lo habitual, pero menor que en varones), 3 puntos (similar a un varón), 4 puntos (superior a lo habitual para un varón). Si no existe vello terminal, puntúa 0.

Tomada de: Yildiz BO, Bolour S, Woods K, Moore A, Azziz R. Visually scoring hirsutism. Hum Reprod Update. 2010;16(1):51-64. Reproducida con permiso de Ricardo Azziz. () 1997 Ricardo Azziz 


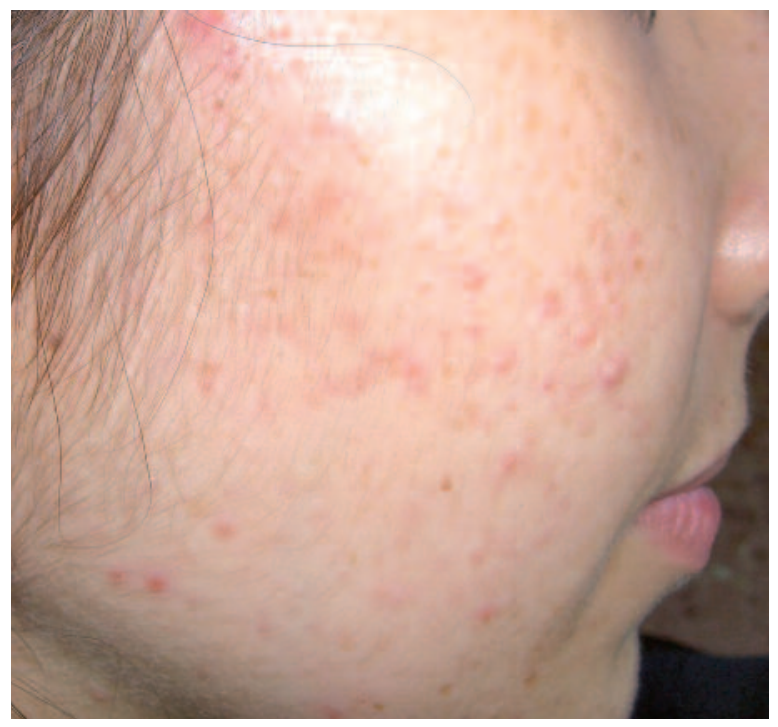

Figura 3. Paciente con síndrome del ovario poliquístico que presenta lesiones inflamatorias en la cara (por gentileza de la Dra. Aurora Guerra-Tapia).

se de forma más tardía y presentar peor respuesta a los tratamientos convencionales que el acné puberal (fig. 3).

En contra de lo que pudiéramos pensar, enfermedades relacionadas con el acné como la hidradenitis supurativa, el quiste pilonidal o la celulitis disecante del cuero cabelludo no ven su prevalencia elevada en las pacientes con SOP.

La patogenia del acné en estas pacientes se debe, fundamentalmente, a los niveles elevados de andrógenos. Los andrógenos ováricos y suprarrenales —-testosterona, DHT, deshidroepiandrosterona (DHEA), sulfato de deshidroepiandrosterona (SDHEA) y androstenodiona- se unen al receptor de andrógenos de la unidad pilosebácea, provocando un aumento del tamaño de la glándula, incrementando la producción de sebo e interfiriendo en el recambio y la queratinización epitelial habitual, lo cual favorece la proliferación de Propionibacterium acnes ${ }^{8}$ y la aparición de comedones y acné inflamatorio. Los niveles sanguíneos de andrógenos no se relacionan con la gravedad del acné ni del hirsutismo, y parecen responder más a una elevación en la sensibilidad del receptor de andrógenos en ciertas loca- lizaciones y a un nivel local elevado de andrógenos, lo que explica por qué muchas mujeres que padecen acné o hirsutismo no presentan ninguna endocrinopatía 9 .

\section{Piel grasa}

Se trata de una característica más prevalente en las pacientes con SOP por el aumento de producción de sebo generado por el hiperandrogenismo, como ya hemos comentado en el punto anterior.

\section{Alopecia}

La alopecia es otro de los signos clínicos del SOP. Se caracteriza por ser una alopecia no cicatricial, que suele respetar la zona occipital y frontal. Los andrógenos - fundamentalmente, la DHT, que se ha generado a partir de la testosterona por efecto de la $5 \alpha$-reductasa a nivel cutáneo- estimulan la conversión del folículo terminal en vello y disminuye el número de folículos en anágeno ${ }^{10}$.

La alopecia no es tan frecuente en las pacientes con SOP como otros síntomas dermatológicos, por lo que, ante su diagnóstico, resulta conveniente descartar otras causas de alopecia femenina como pueden ser la anemia ferropénica, los trastornos tiroideos o la alopecia areata, entre otras.

\section{Acantosis nigricans}

Es una enfermedad mucocutánea que se caracteriza por la presencia de regiones parcheadas hiperpigmentadas, que típicamente se localizan en la base del cuello, las axilas, la fosa antecubital y las ingles. La acantosis nigricans es muy frecuente en pacientes con resistencia periférica a la insulina; se relaciona con el SOP porque un porcentaje variable de estas pacientes presentarán una resistencia a la insulina y tolerancia alterada a los hidratos de carbono. No obstante, la presencia de acantosis debe hacernos pensar en la resistencia a la insulina más que en el SOP.

Otros trastornos dermatológicos, como la hiperhidrosis axilar, muy relacionados con las hormo- 
nas masculinas no han resultado, sorprendentemente, estar asociadas al SOP.

En resumen, la clínica del SOP es multisistémica; se trata de una endocrinopatía cada vez más frecuente, cuya fisiopatología representa un complejo sistema de mecanismos endocrinológicos interconectados, que afecta a las hormonas sexuales, hipotálamo-hipofisarias y a la insulina, pudiendo generar una gran variedad de síntomas diferentes a nivel cardiovascular, reproductivo, metabólico y dermatológico.

Los dermatólogos deben ser conscientes de lo multisistémica de esta endocrinopatía y se encuentran en un lugar privilegiado para su diagnóstico y tratamiento, ya que muchas veces son los síntomas dermatológicos los que harán consultar a la paciente.

\section{DIAGNÓSTICO}

\section{Historia clínica y exploración}

La historia básica debe interrogar acerca de los ciclos menstruales, descartando que estos sean regulares por la toma de anticonceptivos. El hirsutismo se valorará, por ejemplo, mediante la escala de Ferriman-Gallwey, comentada previamente, siendo conscientes de la variabilidad que existe entre diferentes etnias. El hirsutismo moderado o grave (puntuación > 15) debe hacernos pensar en un hiperandrogenismo. También debemos valorar la presencia o ausencia de acné y su respuesta a tratamientos, si es que ya se han aplicado.

\section{Ecografía ginecológica}

La ecografía no es obligatoria para valorar a pacientes con sospecha de SOP, máxime si se trata de pacientes adolescentes, por la alta frecuencia de ovarios con morfología poliquística que existe en estas pacientes. Sin embargo, la ecografía sí que resulta una técnica útil para valorar a aquellas pacientes que presentan alteraciones menstruales aisladas (sin hiperandrogenismo) o hiperandrogenismo sin alteraciones menstruales, ya que el diagnóstico del SOP según los criterios de
Rotterdam solo podrá realizarse, entonces, en presencia de ovarios con morfología ecográfica poliquística. También resulta de especial utilidad la ecografía transvaginal en la valoración de pacientes con hiperandrogenismo grave o de muy rápida evolución para descartar la presencia de tumores ováricos secretores de andrógenos ${ }^{11,12}$ (fig. 4).
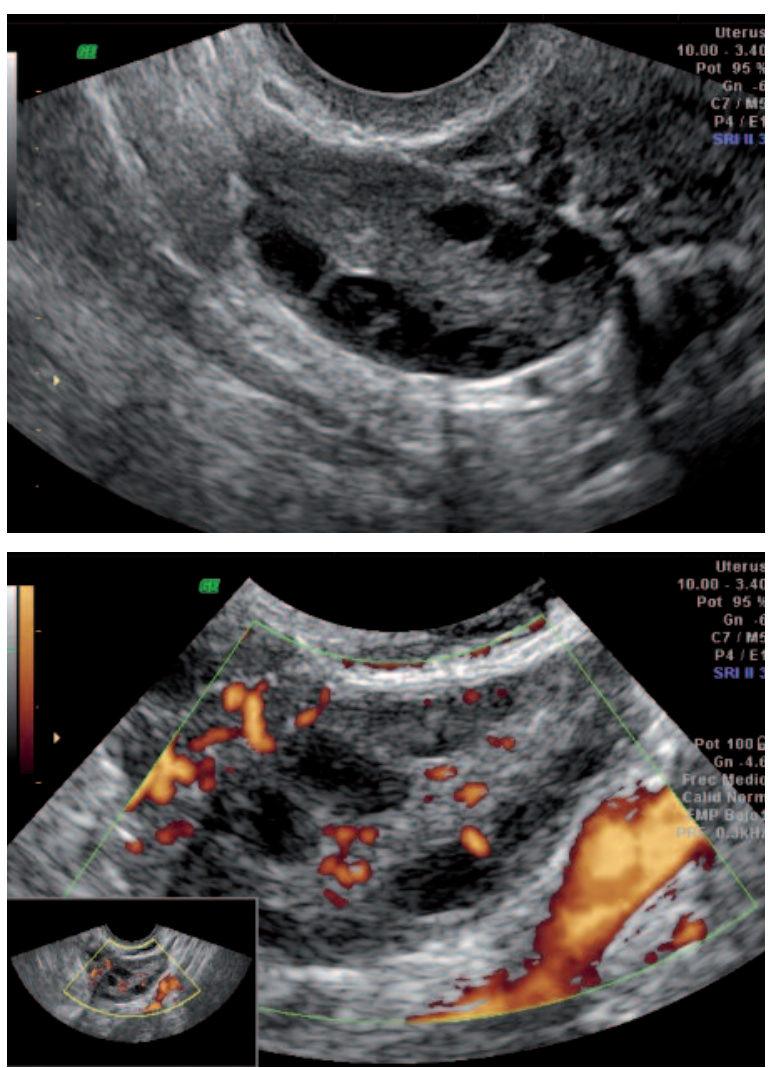

Figura 4. Imagen ecográfica de un ovario poliquístico-vascularización (por gentileza del Dr. José Manuel Puente).

\section{Valoración analítica}

Excluir las enfermedades que puedan causar sintomatología similar al SOP es uno de los objetivos de las pruebas analíticas que realizamos cuando nos encontramos en nuestra actividad clínica con pacientes en las que sospechemos un SOP. No obstante, hay que ser conscientes de la baja prevalencia de ciertas enfermedades y de que las pruebas analíticas tienen un coste económico, 
por lo que, siendo los recursos limitados, debemos intentar ser selectivos a la hora de su petición. No existen artículos publicados acerca de la relación de coste-utilidad de las diferentes pruebas analíticas en estas pacientes, de modo que la medicina basada en la experiencia y el sentido común se convierte aquí en nuestra herramienta para decidir.

Una buena aproximación en este grupo de pacientes sería realizar una analítica inicial en la que valorásemos los niveles de andrógenos. Analíticamente, el hiperandrogenismo es preferible valorarlo por la mañana y, a poder ser, durante la primera fase del ciclo (día 3-10 posmenstrual) mediante un análisis de testosterona libre, ya que, a nivel individual, es el marcador analítico más sensible para diagnosticar el hiperandrogenismo. Como hemos visto, la síntesis elevada de testosterona por parte de las células de la teca y el descenso de SHBG producen un aumento de la testosterona libre (forma activa) en estas pacientes. En centros en los que no se pueda calcular de forma fiable la testosterona libre, puede ser suficiente con calcular la testosterona total.

El nivel superior de testosterona total en adultas es de entre 40 y $60 \mathrm{ng} / \mathrm{dl}$. Las pacientes con SOP pueden tener niveles de entre 30 y $150 \mathrm{ng} / \mathrm{dl}$. Los niveles por encima de los $200 \mathrm{ng} / \mathrm{dl}$ deben hacernos descartar la presencia de un tumor secretor de andrógenos.

Además de estos niveles, podría ser útil en una primera analítica valorar los niveles de tirotropina (TSH) y prolactina, así como los de FSH y LH. Si la paciente es obesa o hipertensa o presenta clínica que nos haga sospecharlo, podemos añadir los niveles de cortisol para descartar la presencia de un síndrome de Cushing. Cuando nos encontramos niveles elevados de andrógenos - especialmente, en pacientes jóvenes-, puede también ser recomendable realizar una determinación de 17-hidroxiprogesterona para descartar la existencia de una hiperplasia suprarrenal congénita. En pacientes en las que sospechemos una acromegalia, la determinación del factor de cre- cimiento insulinoide de tipo 1 (IGF-1) resulta útil para su diagnóstico.

Muchos autores y guías, como las del American College of Obstetricians and Gynecologists (ACOG), la American Association of Clinical Endocrinologists (AACE) o la American Diabetes Association (ADA), recomiendan, además, ante el diagnóstico de SOP - en especial, si se trata de pacientes obesas o con otros factores de riesgo-, descartar la presencia de DM2 o de intolerancia a los hidratos de carbono mediante una sobrecarga oral de glucosa $(75 \mathrm{~g})^{13-15}$. Además, en estas pacientes, resulta aconsejable medir en una primera visita el peso y la talla para calcular el índice de masa corporal (IMC), la presión arterial y realizar junto a la analítica solicitada un estudio del perfil lipídico.

\section{TRATAMIENTO}

\section{Cambios en el estilo de vida}

La pérdida de peso representa la primera línea de tratamiento de las pacientes obesas con SOP. Se recomienda perder un 5-10\% de la masa en el caso de pacientes con sobrepeso u obesidad, y no ganar peso en aquellas pacientes con normopeso.

En dicho objetivo, puede ser útil la restricción calórica, siguiendo una dieta equilibrada, intentando reducir entre 500 y $1000 \mathrm{kcal}^{16}$ diarias.

El ejercicio físico está recomendado en estas pacientes; si bien no existen prácticamente ensayos clínicos aleatorizados, hay evidencia de los efectos beneficiosos del ejercicio en las pacientes con SOP, incluso aunque no exista una pérdida de peso significativa. Practicar ejercicio físico tres días a la semana ha demostrado disminuir el IMC, la circunferencia abdominal y la resistencia a la insulina, y mejorar el consumo de oxígeno y el perfil lipídico ${ }^{17,18}$.

La pérdida de un $5 \%$ de la masa corporal podría mejorar el hirsutismo hasta en un $30 \%$ de las pacientes, aunque una revisión Cochrane no demostró mejoría en el hirsutismo tras los cam- 
bios dietéticos ${ }^{19}$. No obstante, por todo lo expuesto anteriormente y por la inocuidad de la intervención, los cambios en el estilo de vida se recomiendan en todas aquellas pacientes con SOP con sobrepeso u obesidad.

\section{Terapias tópicas}

\section{Eliminación de vello}

Puede realizarse de corta duración mediante cera, sustancias químicas o afeitado, o de larga duración mediante electrólisis, láser o luz pulsada de alta intensidad. No existen estudios que comparen si la terapia con láser resulta igual de efectiva en pacientes con SOP que en la población general. Sin embargo, sí que se ha demostrado que la eliminación de vello, en especial, aquella de larga duración (hoy en día, muy frecuentemente mediante láser), resulta muy útil en las pacientes con SOP que presentan hirsutismo, mejorando la percepción emocional de la enfermedad y la calidad de vida de las pacientes.

\section{Hidrocloruro de eflornitina}

Esta sustancia, en su preparación al 13,9\%, actúa como un inhibidor permanente de la ornitinadescarboxilasa, enzima necesaria para el crecimiento y diferenciación de las células del folículo piloso. Esta acción se revierte a las 6-8 semanas del cese de su aplicación. Por sus posibles efectos sistémicos, se debe aplicar en pequeñas áreas de la piel, por lo que suele reservarse para el hirsutismo facial.

\section{Minoxidil}

La aplicación tópica de minoxidil (al 2 o al $5 \%$ ) promueve el crecimiento del cabello; constituye, así, uno de los pilares del tratamiento de la alopecia en pacientes con SOP. Algunos estudios han demostrado una mayor eficacia del minoxidil al $5 \%$, mientras que otros no encontraron diferencias significativas entre ambos tratamientos, y el uso del minoxidil al $5 \%$ se asocia a un leve incre- mento de los efectos secundarios, como puede ser la hipertricosis facial ${ }^{20}$.

\section{Anticonceptivos hormonales combinados}

La terapia con anticonceptivos hormonales combinados (ACO) constituye una de las herramientas más útiles en el tratamiento de las pacientes con SOP, máxime en aquellas que presentan sintomatología dermatológica, en quienes no solo corrige las irregularidades menstruales, sino que también mejora las manifestaciones dermatológicas del SOP. Antes del uso de ACO, debemos ser conscientes de los efectos metabólicos que pueden tener, así como de sus contraindicaciones absolutas y relativas y de sus efectos secundarios, algunos de ellos, como la cefalea, bastante frecuentes.

De entre los diferentes ACO existentes en el mercado, destacaremos aquellos cuyo principio activo es el etinilestradiol como estrógeno y, como gestágeno (fig. 5), un derivado de la 19-nortestosterona, en especial, los más modernos como el desogestrel, el norgestimato o el gestodeno. Existen derivados más antiguos como el levonorgestrel o el norgestrel, entre otros, que poseen una mayor actividad androgénica, pero que pueden ser igualmente empleados por no haberse demostrado menos efectivos. Todos los fármacos de este grupo pueden ser utilizados, y mejoran el perfil androgénico por tres motivos: el etinilestradiol estimula la síntesis de SHBG hepática, el gestágeno disminuye la secreción de LH y, con ello, la síntesis de andrógenos a nivel de la teca ovárica y, asimismo, como gestágeno, compite con la testosterona por el receptor de andrógenos y a nivel de la $5 \alpha$-reductasa.

Los ACO pueden mejorar la puntuación de hirsutismo en alrededor de 8 puntos, alcanzándose su efecto máximo a los 6-9 meses de tratamiento ${ }^{21}$. Según una revisión Cochrane del año 2012, el uso de ACO reduce las lesiones faciales y la gravedad tanto objetiva como subjetiva del acné, comparado con placebo. 
\begin{tabular}{l|l} 
artículo de revisión & El ovario poliquístico y las enfermedades dermatológicas
\end{tabular}

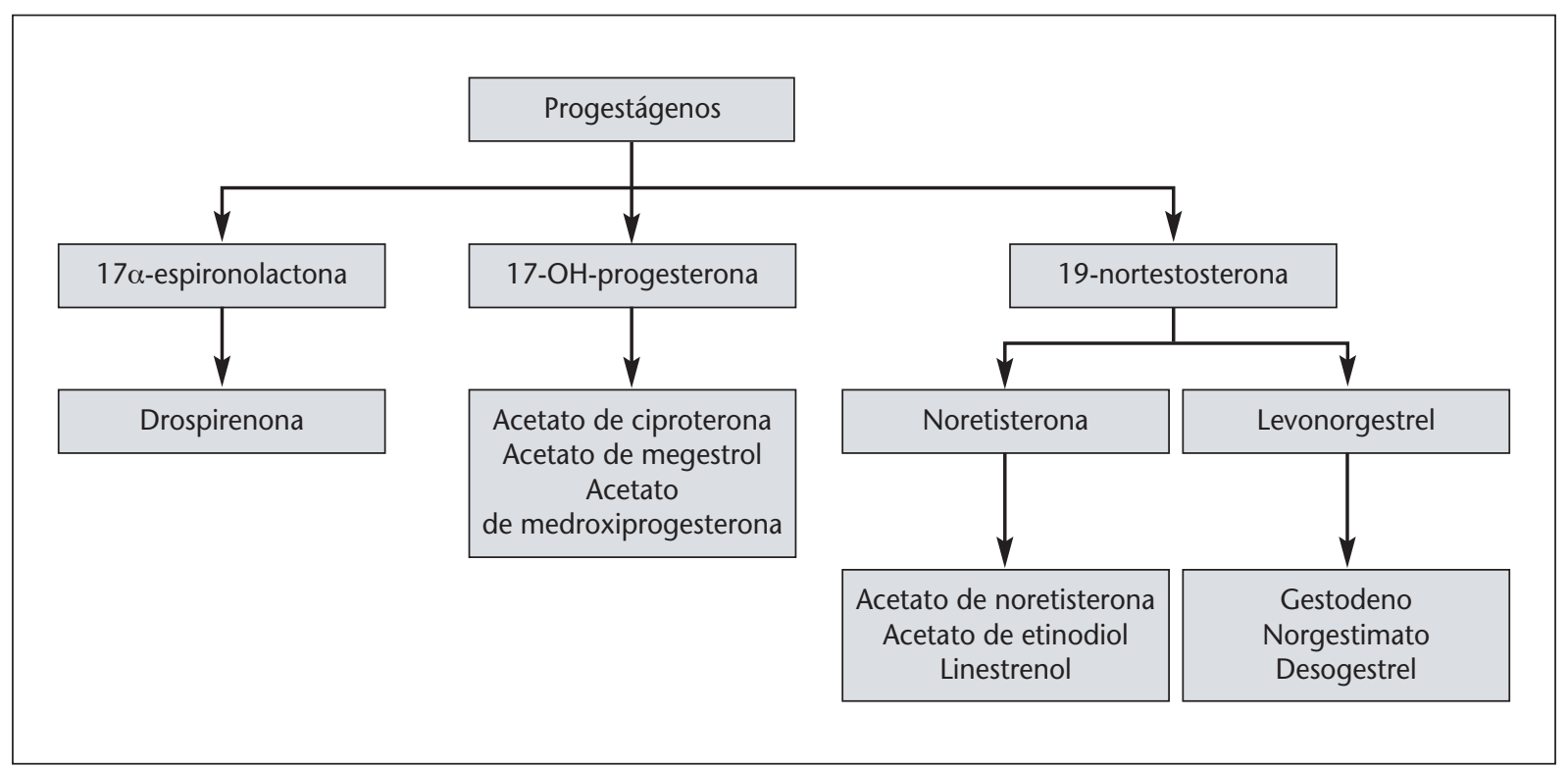

Figura 5. Clasificación de los progestágenos más habituales.

El segundo grupo de anticonceptivos de gran utilidad en las pacientes con SOP son aquellos cuyo estrógeno es el etinilestradiol y su gestágeno lo constituye uno de los gestágenos con perfil antiandrogénico como el acetato de ciproterona o la drospirenona. La drospirenona tiene, además de un perfil antiandrogénico, actividad mineralocorticoidea. El riesgo trombogénico (venoso o pulmonar) de los ACO que contienen acetato de ciproterona o drospirenona es entre una y tres veces mayor que con los gestágenos con menor riesgo trombogénico, como el levonorgestrel. No obstante, el riesgo sigue siendo muy bajo: el riesgo absoluto de tromboembolia venosa por el uso de ACO con drospirenona (25-135 eventos/100000 mujeres-año) es ligeramente superior al de las pacientes que toman ACO con levonorgestrel (692 eventos/100 000 mujeres-año) ${ }^{22}$.

Los ACO con gestágenos con perfil antiandrogénico —en especial, el acetato de ciproteronason efectivos para tratar a las pacientes con hirsutismo, y resultan más eficaces que los ACO con otros gestágenos no antiandrogénicos y que otros fármacos utilizados en el tratamiento del hirsutismo como la espironolactona, el ketoconazol, la flutamida o la finasterida. También son efectivos en el tratamiento del acné y, en varios estudios, han demostrado ser más eficaces que otros $\mathrm{ACO}^{23,24}$.

\section{Fármacos no anticonceptivos con perfil antiandrogénico}

Una revisión sistemática de ensayos clínicos aleatorizados ${ }^{25}$ encontró que el tratamiento con fármacos antiandrogénicos se asociaba a una reducción del hirsutismo de 3,9 puntos en la escala de Ferriman-Gallwey modificada. Algunos estudios sugieren que los antiandrógenos en monoterapia pueden ser superiores a los ACO para el tratamiento del hirsutismo, sin embargo, en combinación, ambos tipos de fármacos podrían ser más eficaces que de forma aislada, por su efecto sinérgico; por ejemplo, la espironolactona asociada a un ACO. Respecto a la eficacia de estos fármacos en el tratamiento del acné en pacientes con SOP, todos ellos parecen aportar una mejoría de los síntomas. La espironolactona y la finasterida han resultado también eficaces en el tratamiento de la alopecia. 
Todos los fármacos antiandrogénicos son teratogénicos, por lo que, durante su uso, debemos asegurarnos de la correcta anticoncepción de la paciente.

\section{Flutamida}

La flutamida es un potente fármaco antiandrogénico, utilizado en el tratamiento del cáncer de próstata. Su uso en el tratamiento del hirsutismo es muy excepcional, ya que se trata de un fármaco de coste elevado y con unos efectos secundarios potencialmente peligrosos, fundamentalmente, por su hepatotoxicidad.

\section{Espironolactona}

Se trata de un diurético ahorrador de potasio que, además de ser un antagonista de la aldosterona, inhibe de forma competitiva la $5 \alpha$-reductasa y el receptor de andrógenos. Uno de sus potenciales, aunque infrecuentes, efectos secundarios es la hiperpotasemia, por lo que, al iniciar su uso o tras la subida de dosis, se recomienda realizar analíticas con determinación de iones para poder diagnosticarla si se produjese. La espironolactona puede producir también ginecomastia y, en algunos estudios, se ha relacionado su uso con un aumento del riesgo de cáncer de mama, sin embargo, otros estudios no han encontrado esta asociación ${ }^{26}$.

\section{Finasterida}

Es un fármaco inhibidor de la $5 \alpha$-reductasa, que impide la conversión de testosterona a su forma más activa, la DHT. Puede ser utilizada en dosis de $5 \mathrm{mg}$ /día o de 2,5 mg/día, con la ventaja de que la menor dosis presenta menos efectos secundarios, menor coste y una efectividad similar.

\section{Otros fármacos con perfil antiandrogénico} En dosis de $400 \mathrm{mg} /$ día, el antifúngico ketoconazol presenta también acción antiandrogénica. Entre sus efectos secundarios, destaca la hipertransaminasemia, que, aunque leve, puede afectar hasta al 5-10\% de pacientes.
La leuprorelina es un análogo de la GnRH; aunque al comienzo del tratamiento provoca un efecto de estimulación inicial (flare up) transitorio, su uso continuado produce una regulación por disminución (down-regulation) a nivel hipofisario, lo que se traduce en una reducción de la síntesis de LH y FSH, provocando, así, un descenso de los niveles de andrógenos.

En dosis de $300 \mathrm{mg}$ /día, el antihistamínico cimetidina podría ser eficaz en el tratamiento del acné y el hirsutismo, aunque su uso con este fin resulta casi anecdótico.

La metformina aumenta la sensibilidad a la insulina y, de forma indirecta, reduce la producción de andrógenos en la teca ovárica. Algunos estudios sugieren que puede aportar mejoría en el hirsutismo, aunque otros no lo han demostrado. Respecto a la mejoría del acné o la alopecia, no existen datos consistentes. La metformina mejora el ciclo menstrual y la fertilidad, disminuye la tasa de aborto y el riesgo de DM2 y mejora el perfil lipídico ${ }^{27}$.

La isotretinoína, en algunos estudios, ha demostrado disminuir la actividad de la $5 \alpha$-reductasa. Es útil en el tratamiento del acné en el SOP, pero no tiene eficacia en el tratamiento del hirsutismo ni de la alopecia, la cual puede incluso empeorar $^{28}$.

\section{Recomendaciones terapéuticas ${ }^{21}$}

\section{Hirsutismo}

El tratamiento del hirsutismo en la paciente con SOP puede iniciarse con un ACO, en especial, aquellos con bajo perfil androgénico o con perfil antiandrogénico. Posteriormente, se puede añadir espironolactona si se precisa, empezando con $50 \mathrm{mg}$ diarios y elevando la dosis si no resulta suficientemente efectiva; las dosis de 50-100 mg parecen presentar una eficacia similar a los $200 \mathrm{mg}$ y menor cantidad de efectos adversos. El tratamiento con un ACO que contenga drospirenona equivale a $25 \mathrm{mg}$ de espironolactona. 


\section{Acné}

Resulta recomendable iniciar el tratamiento del acné con terapias tópicas. Si estas no resultan efectivas, podemos iniciar un tratamiento con un ACO con perfil antiandrogénico como aquellos que contienen acetato de ciproterona o drospireno$n^{29-31}$. Si a los 3-6 meses de iniciado el tratamiento no resulta suficientemente efectivo, se puede añadir espironolactona, con las mismas consideraciones que las expuestas anteriormente. Se puede ofrecer isotretinoína o antibióticos como segunda línea del tratamiento del acné de estas pacientes.

\section{BIBLIOGRAFÍA}

1. Stein Fl, Leventhal ML. Amenorrhea associated with bilateral polycystic ovaries. Am J Obstet Gynecol. 1935;29:181-91.

2. Azziz R, Woods KS, Reyna R, Key TJ, Knochenhauer ES, Yildiz $B O$. The prevalence and features of the polycystic ovary syndrome in an unselected population. J Clin Endocrinol Metab. 2004;89(6):2745-9.

3. Franks S. Polycystic ovary syndrome. N Engl J Med. 1995; 333(13):853-61.

4. Baskind NE, Balen AH. Hypotahalamic-pituitary, ovarina and adrenal contributions to polycystic ovary syndrome. Best Pract Res Clin Obstet Gynaecol. 2016;37:80-97.

5. Nelson VL, Qin KN, Rosenfield RL, Wood JR, Penning TM, Legro RS, et al. The biochemical basis for increased testosterone production in theca cells propagated from patients with polycystic ovary syndrome. J Clin Endocrinol Metab. 2001; 86(12):5925-33.

6. Rosenfield RL. Clinical practice. Hirsutism. N Engl J Med. 2005; 353(24):2578-88.

7. Falsetti L, Gambera A, Andrico S, Sartori E. Acne and hirsutism in polycystic ovary syndrome: clinical, endocrine-metabolic and ultrasonographic differences. Gynecol Endocrinol. 2002;16(4):275-84.

8. Schimdt JB, Spona J, Huber J. Androgen receptor in hirsutism and acne. Gynecol Obset Invest. 1986;22(4):206-11.

9. Deplewski D, Rosenfield RL. Role of hormones in pilosebaceous unit development. Endocr Rev. 2000;21(4):363-92.

10. Cela E, Robertson C, Rush K, Kousta E, White DM, Wilson H, et al. Prevalence of polycystic ovaries in women with androgenetic alopecia. Eur J Endocrinol. 2003;149(5):439-42.

11. Kenigsberg LE, Agarwal C, Sin S, Shifteh K, Isasi CR, Crespi $\mathrm{R}$, et al. Clinical utility of magnetic resonance imaging and ultrasonography for diagnosis of polycystic ovary syndrome in adolescent girls. Fertil Steril. 2015;104(5):1302-9.

12. Dewailly D, Lujan ME, Carmina E, Cedars MI, Laven J, Norman RJ, et al. Definition and significance of polycystic ovarian morphology: a task force report from de Androgen Excess and Polycystic Ovary Syndrome Society. Hum Reprod Update. 2014;20(3):334-52.

13. Fauser BC, Tarlatzis BC, Rebar RW, Legro RS, Balen AH, Lobo $\mathrm{R}$, et al. Consensus on women's health aspects of polycystic ovay syndrome (PCOS): the Amsterdam ESHRE/ASRMSponsored 3rd PCOS Consensus Workshop Group. Fertil Steril. 2012;97(1):28-38.e25.
14. American Association of Clinical Endocrinologists Polycystic Ovary Syndrome Writing Committee. American Association of Clinical Endocrinologists Position Statement on Metabolic and Cardiovascular Consequences of Polycystic Ovary Syndrome. Endocr Pract. 2005;11(2):126-34.

15. Salley KE, Wickham EP, Cheang KI, Essah PA, Karjane NW, Nestler JE. Glucose intolerance in polycystic ovary syndrome. A position statement of the Androgen Excess Society. J Clin Endocrinol Metab. 2007;92(12):4546-56.

16. De Sousa SM, Norman RJ. Metabolic syndrome, diet and exercise. Best Pract Res Clin Obstet Gynaecol. 2016;37:140-51.

17. Vigorito C, Giallauria F, Palomba S, Cascella T, Manguso F, Lucci $R$, et al. Beneficial effects of a three-month structured exercise training program on cardiopulmonary functional capacity in young women with polycystic ovary syndrome. J Clin Endocrinol Metab. 2007;92(4):1379-84.

18. Hutchison SK, Stepto NK, Harrison CL, Moran LJ, Strauss BJ, Teede $\mathrm{HJ}$. Effects of exercise on insulin resistance and body composition in overweight and obese women with and without polycystic ovary syndrome. J Clin Endocrinol Metab. 2011;96(1):E48-56.

19 Moran LJ, Hutchison SK, Norman RJ, Teede HJ. Lifestyle changes in women with polycystic ovary syndrome. Cochrane Database Syst Rev. 2011;(7):CD007506.

20. Lizneva D, Gavrilova-Jordan L, Walker W, Azziz R. Androgen excess: investigations and management. Best Pract Res Clin Obstet Gynaecol. 2016;37:98-118.

21. Buzney E, Sheu J, Buzney C, Reynolds RV. Polycystic ovary syndrome: a review for dermatologists: Part II. J Am Acad Dermatol. 2014;71(5):859.e1-859.e15.

22. Lidegaard $\varnothing$, Milsom I, Geirsson RT, Skejeldestad FE. Hormonal contraception and venous thromboembolism. Acta Obstet Gynecol Scand. 2012;91(7):769-78.

23. Barth JH, Cherry CA, Wojnarowska F, Dawber RP. Cyproterone acetate for severe hirsutism: results of a double-blind doseranging study. Clin Endocrinol (Oxf). 1991;35(1):5-10.

24. Arowojolu AO, Gallo MF, Lopez LM, Grimes DA. Combined oral contraceptive pills for treatment of acne. Cochrane Database Syst Rev. 2012;(7):CD004425.

25. Swiglo BA, Cosma M, Flynn DN, Kurtz DM, Labella ML, Mullan $\mathrm{RJ}$, et al. Clinical review: antiandrogens for the treatment of hirsutism: a systematic review and metaanalyses of randomized controlled trials. J Clin Endocrinol Metab. 2008;93(4): 1153-60.

26. Mackenzie IS, Macdonald TM, Thompson A, Morant S, Wei L. Spironolactone and risk of incident breast cancer in women older than 55 years: retrospective, matched cohort study. BMJ. 2012;345:e4447.

27. Harborne L, Fleming R, Lyall H, Norman J, Sattar N. Descriptive review of the evidence for the use of metformin in polycystic ovary syndrome. Lancet. 2003;361(9372):1894-901.

28. Lowenstein E. Diagnosis and management of the dermatologic manifestations of the polycystic ovary syndrome. Dermatol Ther. 2006;19(4):210-23.

29. Guerra-Tapia A, Sancho Pérez B. Ethinylestradiol/Chlormadi none acetate: dermatological benefits. Am J Clin Dermatol. 2011;12 Suppl 1:3-11.

30. Sancho B, Guerra-Tapia A. Oral contraceptives in dermatology. Actas Dermosifiliogr. 2009;100(6):445-55.

31. Rivera R, Guerra-Tapia A. Management of acne in women over 25 years of age. Actas Dermosifiliogr. 2009;100(1):33-7. 